\title{
Electronic structure and electric polarity of edge-functionalized graphene nanoribbons
}

\author{
Remi Taira, Ayaka Yamanaka*†, and Susumu Okada ${ }^{\ddagger}$ \\ Graduate School of Pure and Applied Sciences, University of Tsukuba, Tsukuba, Ibaraki 305-8571, Japan
}

\begin{abstract}
On the basis of the density functional theory combined with the effective screening medium method, we studied the electronic structure of graphene nanoribbons with zigzag edges, which are terminated by functional groups. The work function of the nanoribbons is sensitive to the functional groups. The edge state inherent in the zigzag edges is robust against edge functionalization. $\mathrm{OH}$ termination causes the injection of electrons into the nearly free electron states situated alongside the nanoribbons, resulting in the formation of free electron channels outside the nanoribbons. We also demonstrated that the polarity of zigzag graphene nanoribbons is controllable by the asymmetrical functionalization of their edges.
\end{abstract}

\section{Introduction}

A honeycomb covalent network of $\mathrm{sp}^{2} \mathrm{C}$ makes graphene a unique material in the fields of composite and electronic devices. ${ }^{1,2)}$ Tightly bound $\sigma$ electrons render a twodimensional hexagonal covalent network with an atom thickness, causing a remarkable mechanical strength. ${ }^{1,2)}$ The mechanically stiff network allows them to be used as promising materials for thermal devices owing to their high thermal conductivity. ${ }^{3-6)}$ In contrast to the $\sigma$ electrons, $\pi$ electrons are distributed normal to the sheet and their distribution is extended throughout the sheet because of the small overlap of their wave functions between adjacent atoms. The bipartite lattice of hexagonally arranged $\mathrm{C}$ atoms leads to a unique electronic structure in $\pi$ electron states: graphene possesses a pair of linear dispersion band at the Fermi level and at the six corners of the Brillouin zone, leading to a remarkable carrier mobility, ${ }^{7-9)}$ which reaches up to 200,000 $\mathrm{cm}^{2} \mathrm{~V}^{-1} \mathrm{~s}^{-1}$, allowing graphene to be an emerging material for high-speed electronic devices. ${ }^{10-14)}$

In addition to the intrinsic unique electronic properties of graphene, graphene ex-

\footnotetext{
${ }^{\dagger}$ Present address: Research Organization for Information Science and Technology (RIST), Shinagawa, Tokyo 140-0001, Japan

${ }^{*}$ E-mail address: yamanaka@rist.or.jp

${ }^{\ddagger}$ E-mail address: sokada@comas.frsc.tsukuba.ac.jp
} 
hibits further variation in electronic structure caused by imposing additional boundary conditions. The porous graphene networks possess flat or narrow dispersion bands at the Fermi level, leading to the magnetic ordering depending on their porous structure. ${ }^{15,16)}$ One-dimensional open boundary conditions cause the formation of the strip structures of graphene with nanometer widths known as graphene nanoribbons. The nanoribbons possess various edge shapes ranging from zigzag to armchair. The energetic stability and electronic structure of graphene nanoribbons are sensitive to the edge atomic arrangement. ${ }^{17-19)}$ Graphene nanoribbons with armchair or near armchair edges are either metals or semiconductors whose band gap asymptotically decreases with increasing width. On the other hand, graphene nanoribbons with zigzag and nearzigzag edges possess half-filled flat dispersion bands at the Fermi level associated with the nonbonding states around the edge atomic sites, leading to spin polarization. Edge functionalization also leads to further variation in electronic structure. ${ }^{2-23)}$ In addition to the edges, the borders between graphene and other two-dimensional materials also lead to border-localized states with nonbonding nature as in the case of edge states. ${ }^{24,25)}$

The edges and surfaces of graphene nanoribbons lead to peculiar extended state whose wave function maximum is located in vacuum where atoms are absent. The dense and atomically flat covalent networks of graphene and graphene nanoribbons induce an attractive potential outside the network in which the unoccupied states are bound outside the atomic network and below the vacuum level. Such states are bound to the surfaces of graphitic networks, while their distribution is extended along the surfaces with free electron nature, thereby being known as the nearly free electron (NFE) states of atom layer materials and the interlayer states of layered materials. ${ }^{26-33)}$ In addition to the surfaces, NFE states also emerge alongside the edges of graphene. ${ }^{34-36)}$ Furthermore, the NFE states are sensitive to external environmental factors, such as external electric field, atom adsorption, functionalization, and electron doping, because of the weak screening in the NFE states arising from their unoccupied nature. ${ }^{35,36)}$ In our previous work, we demonstrated that the hydroxyl functionalization of graphene edges causes the downward shift of the NFE states and forms an electron conducting channel with a free electron mass alongside the hydroxyl edge. ${ }^{23)}$

Since the electronic structures of graphene and its derivatives are sensitive to their geometric structures and the external perturbations, it is mandatory to elucidate in detail their electronic properties for application as constituent materials in the wide area of the current nanotechnology. In particular, edge functionalization is one of key 
procedures used to tailor the geometries, stability, and electronic properties of graphene nanomaterials, because it affects the stability and physical properties of graphene flakes. Thus, in this work, we aim to theoretically investigate the electronic properties of graphene nanoribbons whose edges are symmetrically and asymmetrically terminated by functional groups using the density functional theory (DFT) combined with the effective screening medium (ESM) method. Our calculations on the symmetrically terminated zigzag graphene nanoribbons showed that the work function of the functionalized zigzag edges of graphene depends on the attached functional group. The hydroxylation of the zigzag edges results in a work function smaller than that for the edges terminated by $\mathrm{H}$, ketone, aldehyde, and carboxyl groups, thereby leading to the injection of electrons into the NFE states outside and alongside the edges. By asymmetrically functionalizing graphene nanoribbons, the nanoribbons exhibit an electric polarity whose direction and intensity approximately correspond to the difference between the work functions of functionalized edges.

\section{Methods and Models}

For all calculations, we use $\mathrm{DFT}^{37,38)}$ implemented into the simulation tool for atom technology (STATE). ${ }^{39)}$ The exchange correlation potentials among the interacting electrons are described by the local density approximation with the Perdew-Zunger functional form fitted to the Quantum Monte Carlo results for a homogeneous electron gas . ${ }^{40,41)}$ The ultrasoft pseudopotentials generated using the Vanderbilt scheme were used to describe electron-ion interactions. ${ }^{42)}$ Valence wave functions and deficit charge densities were expanded in terms of the plane wave basis set with cutoff energies of 25 and 225 Ry, respectively. To exclude unphysical dipole-dipole interaction with the periodic imaging cells for the asymmetrically functionalized graphene nanoribbons, we adopted the ESM method ${ }^{43)}$ within the frame work of the DFT with the plane wave basis set. Here, we set the effective screening mediums alongside edges of the nanoribbons with a relative permittivity of 1 , which mimics an open boundary condition at two cell boundaries with respect to the electrostatic interaction. The atomic structures were fully optimized until the force acting on each atom was less than $5 \mathrm{mRy} / \AA$ under the fixed lattice parameter of $0.49 \mathrm{~nm}$ along the direction of nanoribbons, which corresponds to the double periodicity of that of the zigzag nanoribbons. Integration over the one-dimensional Brillouin zone was carried out by equidistant $k$-point sampling, in which $4 k$-points were taken along the nanoribbon direction, which corresponds to 8 


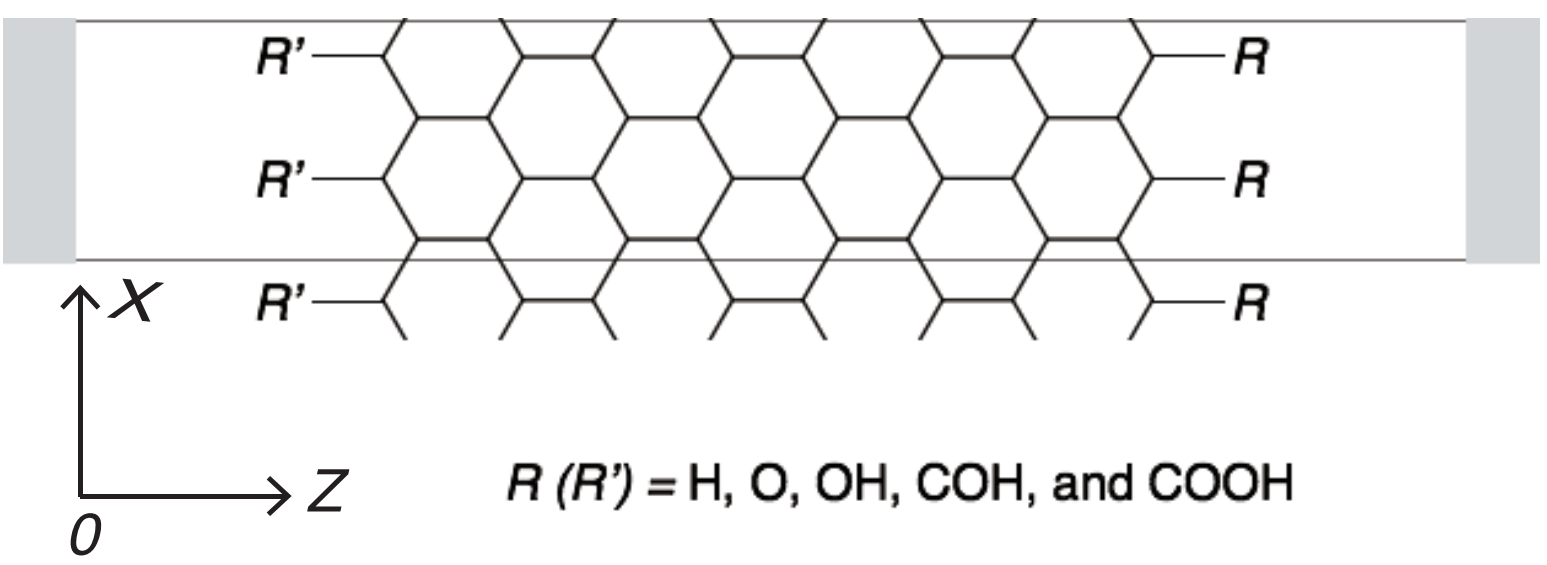

Fig. 1. Structural model of symmetrically and asymmetrically functionalized graphene nanoribbons with zigzag edges. $R\left(R^{\prime}\right)$ indicate $\mathrm{H}$, ketone $(\mathrm{O})$, hydroxyl $(\mathrm{OH})$, aldehyde $(\mathrm{COH})$, and carboxyl $(\mathrm{COOH})$ groups. The rectangle denotes the unit cell adopted in this work. Gray rectangle indicate the cell boundaries simulated by ESM with the vacuum permittivity $\varepsilon_{0}$.

$k$-point sampling for the primitive cell of graphene, which gives sufficient convergence for both geometric and electronic structures.

In the present work, we consider zigzag graphene nanoribbons with a width of $1.56 \mathrm{~nm}$ whose edge atomic sites are symmetrically and asymmetrically terminated by $\mathrm{H}$, ketone, hydroxyl, aldehyde, and carboxyl groups as representative functional groups (Fig. 1). For the aldehyde and carboxyl groups, to avoid the steric hindrance between adjacent edge atomic sites, edge $\mathrm{C}$ sites are alternately terminated by these functional groups and hydrogen atoms. To simulate an isolated graphene nanoribbon, each graphene nanoribbon is separated by its periodic images by 6 and $10 \AA$ vacuum spacing normal and lateral directions, respectively.

\section{Results and Discussion}

Figure 2 shows the electrostatic potential of zigzag graphene nanoribbons whose edges are symmetrically functionalized by $\mathrm{H}$, ketone, hydroxyl, aldehyde, and carboxyl groups. The calculated work functions of the functionalized zigzag edges are 3.85, 8.00, 2.42, 6.58, and $4.80 \mathrm{~V}$ for $\mathrm{H}$, ketone, hydroxyl, aldehyde, and carboxyl groups, respectively, which indicate that the work function of the edge is sensitive to the functional group. Among the functional groups studied here, the hydroxyl edge has the smallest work function. while the ketone edge has the largest work function. In addition to the work function, the potential profile in the nanoribbons also depends on the functional group. Throughout the nanoribbon functionalized by $\mathrm{H}$, the electrostatic potential of $\mathrm{C}$ atomic 

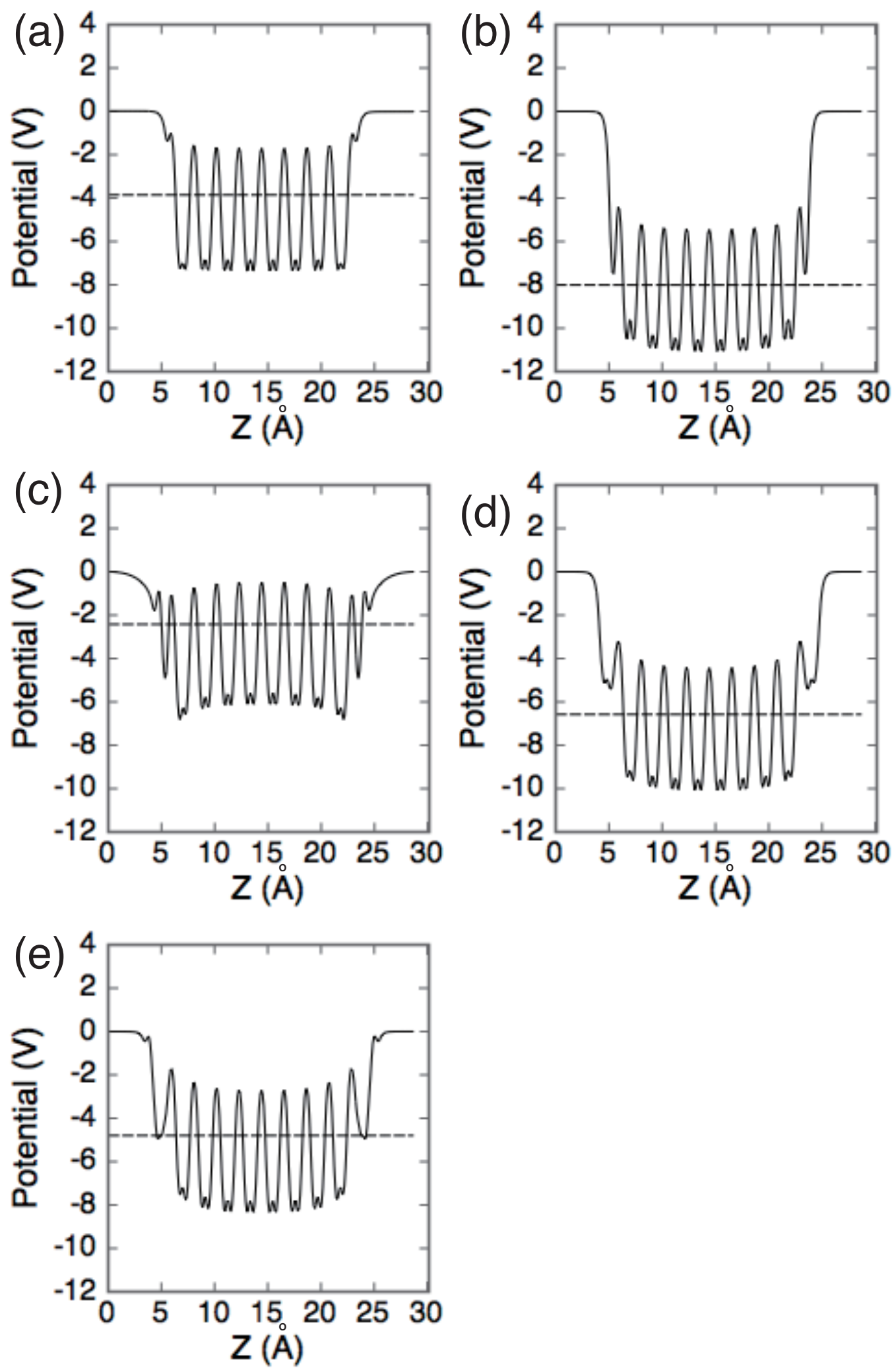

Fig. 2. Plane-averaged electrostatic potential across the graphene nanoribbons with zigzag edges symmetrically functionalized by (a) H, (b) ketone, (c) hydroxyl, (d) aldehyde, and (e) carboxyl groups. Horizontal dotted lines indicate the Fermi level energy. The horizontal axis (z-axis) corresponds to the direction across the nanoribbons as shown in Fig. 1. 
(a)

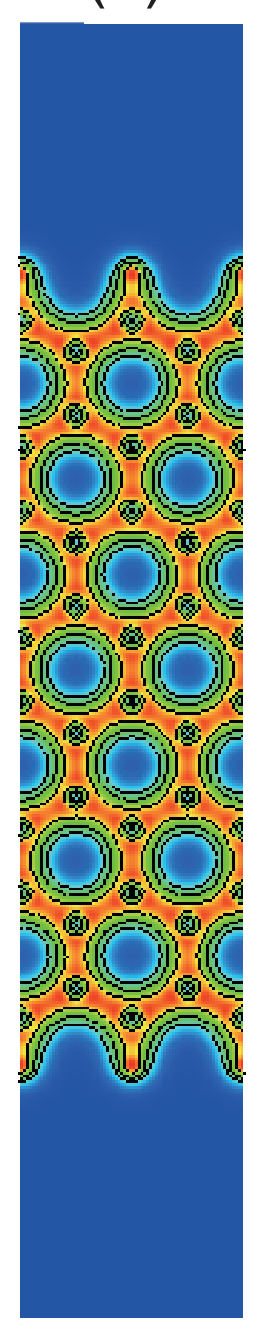

(b)
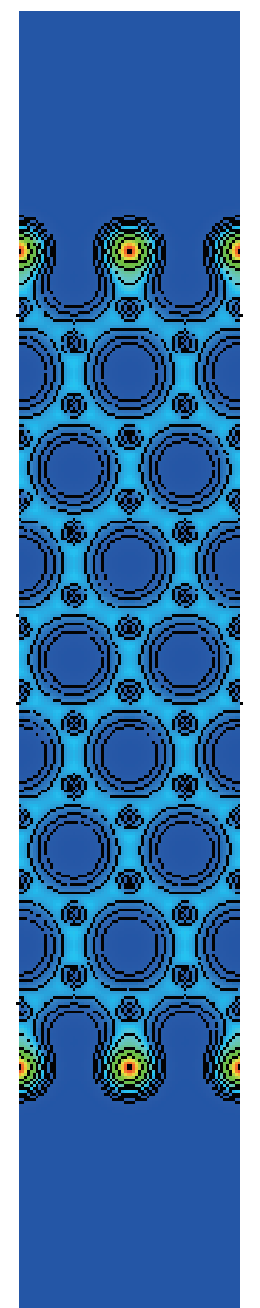

(c)
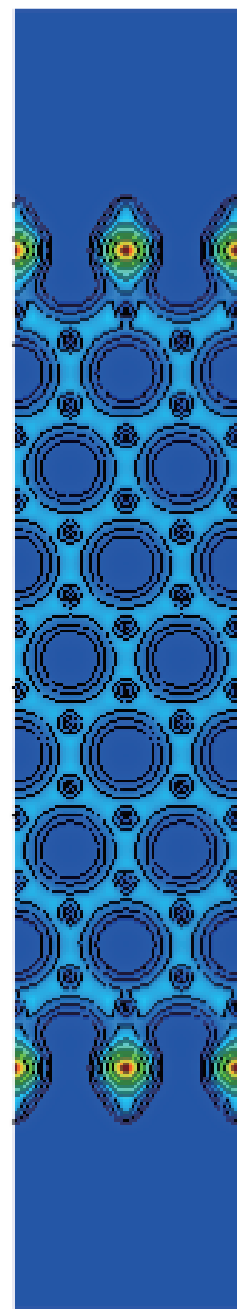

(d)
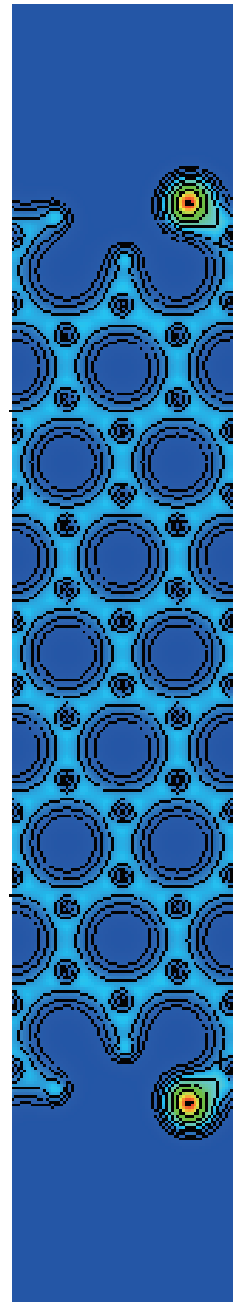

(e)

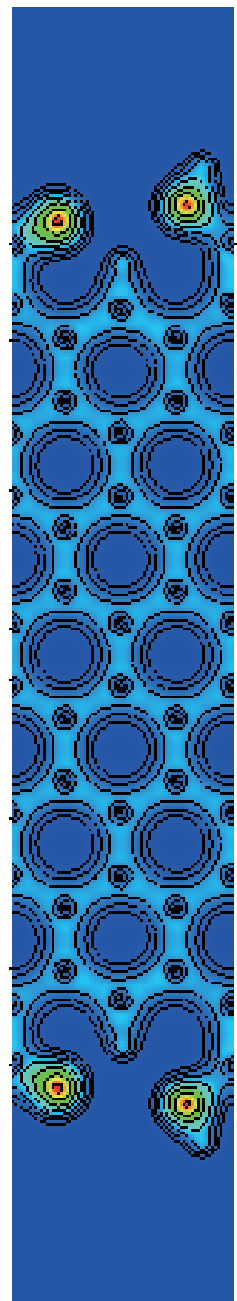

Fig. 3. (Color online) Contour (color) plots of the valence charge densities of the graphene nanoribbons with zigzag edges symmetrically functionalized by (a) H, (b) ketone, (c) hydroxyl, (d) aldehyde, and (e) carboxyl groups. The red, yellow, and blue colors correspond to the high, moderate, and low electron densities, respectively.

sites is approximately constant. On the other hand, the potential of $\mathrm{C}$ atoms in the central region is higher or lower than that of edge $\mathrm{C}$ sites for hydroxyl or ketone, aldehyde, and carboxyl groups. The potential modulation inside the nanoribbon is ascribed to the deficit electrons at edge $\mathrm{C}$ atoms functionalized by the hydroxyl group and the surplus electrons at edge $\mathrm{C}$ atoms functionalized by ketone, aldehyde, and carboxyl groups.

Figure 3 shows the contour plots of the valence charge densities of graphene nanorib- 
bons with functionalized zigzag edges. By analyzing the charge density around the edge $\mathrm{C}$ atomic sites, we found that the electron density around the edge $\mathrm{C}$ atomic sites functionalized by the hydroxyl group is slightly lower than that around the $\mathrm{C}$ atoms situated inner region of the nanoribbon. In the cases of the nanoribbons terminated by the ketone and aldehyde groups, the electron density on the heterobond is higher than that that on $\mathrm{C}-\mathrm{C}$ bonds, leading to the modulation of the electrostatic potential of $\mathrm{C}$ atoms across the nanoribbons.

The electronic energy band and squared wave function at the Fermi level and the $\Gamma$ point are depicted in Fig. 4. As shown in Fig. 4, the detailed electronic structure of edge functionalized graphene nanoribbons depends on the attached functional group. On the other hand, except the nanoribbon functionalized by the ketone group, we find that the nanoribbons possess a pair of flat dispersion bands around the $\Gamma$ point. The wave function distribution indicates that the states are mainly distributed at the edge $\mathrm{C}$ with nonbonding $\pi$ nature [ $\alpha$ and $\beta$ states in Figs. 4(a), 4(c), 4(d), and 4(e)]. Thus, these states are characterized as the edge states as in the case of the nanoribbon with hydrogenated zigzag edges. In contrast, the nanoribbon with ketone edges does not possess the flat band associated with the edge states, but instead two dispersive bands cross the Fermi level with the antibonding $p$ nature of $\mathrm{O}$ atoms parallel to the nanoribbon $[\alpha$ and $\beta$ states in Fig. $4(\mathrm{~b})]$.

For the nanoribbon with aldehyde and carboxyl edges, the electronic structure near the Fermi level is approximately the same as that of the nanoribbon with hydrogenated edges. In these cases, a small dispersion band additionally emerges just below the partial flat bands associated with the edge states. The states is distributed on $\mathrm{O}$ atoms with $p$ nature [the $\gamma$ states in Figs. 4(d) and 4(e)]. In contrast, for the nanoribbon with the hydroxyl edges, an additional parabolic band emerges at the $\Gamma$ point and crosses the Fermi level. The state has its maximum amplitude in the vacuum region outside the nanoribbon which is extended alongside the edges [the $\gamma$ state in Fig. 4(c)]. Thus, the parabolic band is classified as the NEF state. The NFE states on and alongside the graphene nanoribbon with hydrogenated edges are unoccupied states located 2-3 eV above the Dirac point. The state shifts downward owing to the surface/edge dipole moment induced by the external electric field or atomic/molecular doping. Thus, the hydroxylation of the graphene nanoribbon induces the dipole around the edge atomic sites leading to the effective electric field outside the nanoribbon, which causes the substantial downward shift of the NFE states without the external field or foreign 

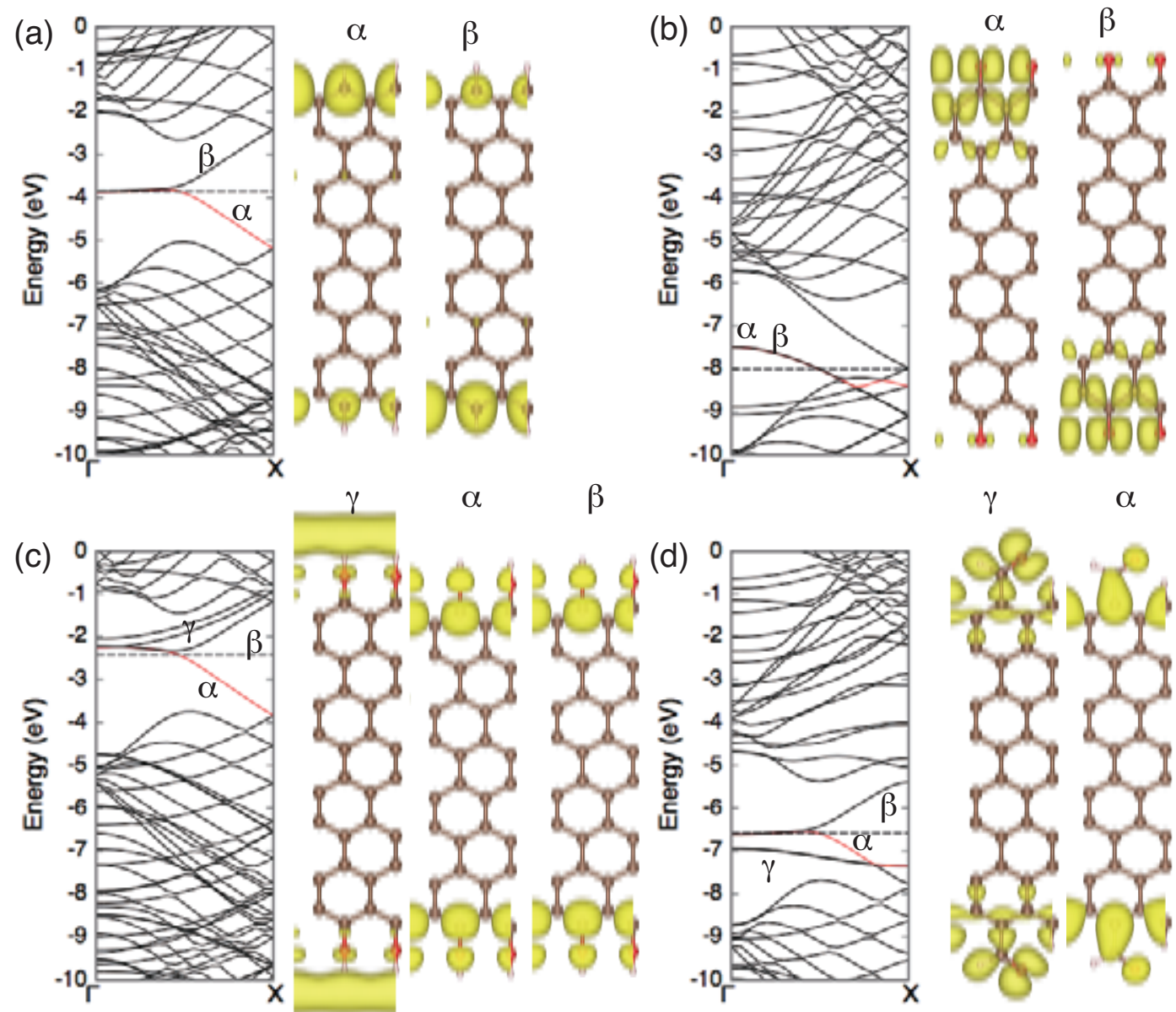

$\alpha \quad \beta$

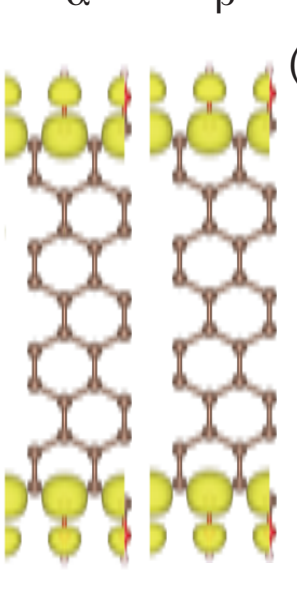

(d)
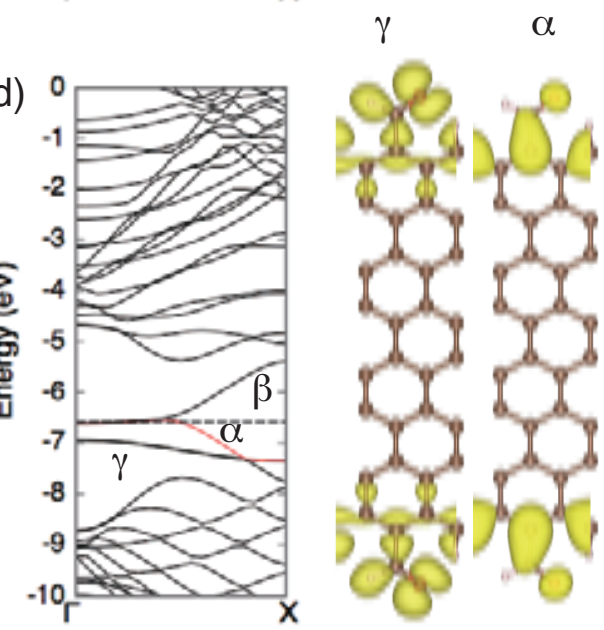

$\beta$

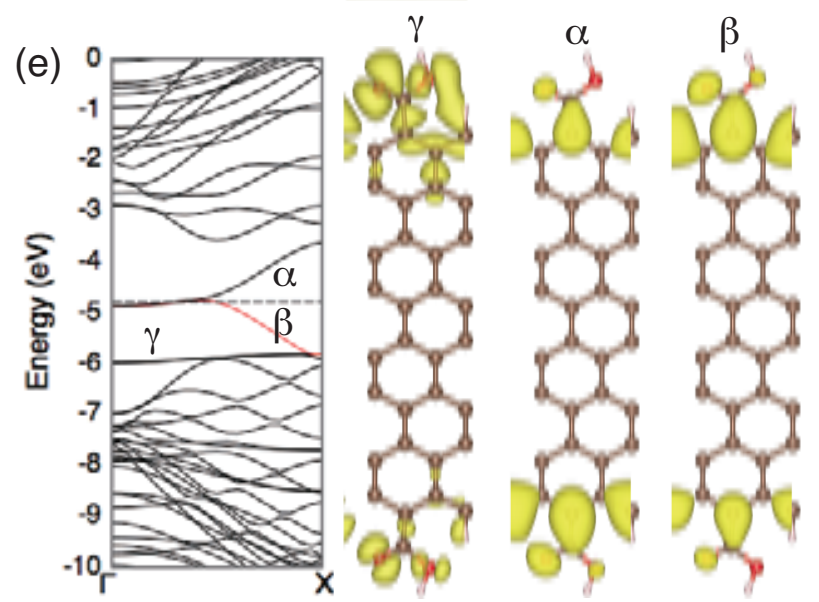

Fig. 4. (Color online) Electronic energy band and squared wave function crossing the Fermi level of the graphene nanoribbons with zigzag edges symmetrically functionalized by (a) H, (b) ketone, (c) hydroxyl, (d) aldehyde, and (e) carboxyl groups. The red curve indicate the highest branch of the valence band. Horizontal dotted lines denote the Fermi level energy. The labels of the wave functions correspond to those of the electron states at the $\Gamma$ point. Blown, red, and white circles denote $\mathrm{C}$, O, and $\mathrm{H}$ atoms, respectively. 
Table I. Electrostatic potential difference $\Delta V(\mathrm{eV})$ between two edges of asymmetrically functionalized graphene nanoribbons $R^{\prime} / R$ where $R\left(R^{\prime}\right)$ corresponds to $\mathrm{H}, \mathrm{O}, \mathrm{OH}, \mathrm{COH}$, and $\mathrm{COOH}$ groups attached to the left (right) edges. The difference is evaluated as $\Delta V=E_{V}^{R}-E_{V}^{R^{\prime}}$, where $E_{V}^{R}$ and $E_{V}^{R^{\prime}}$ are the energies of the vacuum levels of graphene edges functionalized by $R$ and $R^{\prime}$, respectively. The energies are measured from that of the edge terminated by $R^{\prime}$.

\begin{tabular}{ccccc}
\hline $\mathrm{H} / \mathrm{O}$ & $\mathrm{H} / \mathrm{OH}$ & $\mathrm{H} / \mathrm{COH}$ & $\mathrm{H} / \mathrm{COOH}$ & $\mathrm{O} / \mathrm{OH}$ \\
4.31 & -1.90 & 2.73 & 1.09 & -6.35 \\
\hline $\mathrm{O} / \mathrm{COH}$ & $\mathrm{O} / \mathrm{COOH}$ & $\mathrm{OH} / \mathrm{COH}$ & $\mathrm{OH} / \mathrm{COOH}$ & $\mathrm{COH} / \mathrm{COOH}$ \\
-1.60 & -3.30 & 4.65 & 1.78 & -1.56 \\
\hline
\end{tabular}

atoms.

The work function of the graphene edges depends on the functional group attached to the edges, thereby inducing the polarity normal to the nanoribbon direction by the asymmetric functionalization of zigzag graphene nanoribbons. Figure 5 shows the plane averaged electrostatic potential across the graphene nanoribbon whose edges are asymmetrically functionalized by $\mathrm{H}$, ketone, hydroxyl, aldehyde, and carboxyl groups. In all cases, the potential indicates asymmetric nature. The potential difference between two edges depend on the functional groups. The electrostatic potential at the edges functionalized by the hydroxyl group is lower than those at the edges functionalized by the other functional groups. Accordingly, the electrostatic potential of $\mathrm{C}$ atoms attached by the hydroxyl group is deeper than that of the $\mathrm{C}$ atom inner region of the nanoribbons. The results indicate that the asymmetrical functionalization of graphene nanoribbons induces the polarity across the nanoribbons, which is controllable by selecting the functional groups. The calculated potential difference is shown in Table I. The potential sign and difference between two functionalized edges approximately correspond to the work function difference between two edges.

\section{Conclusion}

We studied the geometric and electronic structures of graphene nanoribbons with zigzag edges, which are symmetrically and asymmetrically terminated by $\mathrm{H}$, ketone, hydroxyl, aldehyde, and carboxyl groups, using the first-principles total-energy calculations within the framework of DFT. Our calculations show that the work function and electronic structure of graphene edges are sensitive to the functional group species. The work function of the edge functionalized by hydroxyl groups is the smallest among the edges 
(a)

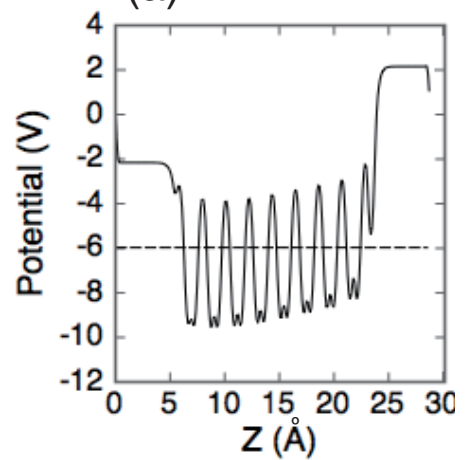

(d)

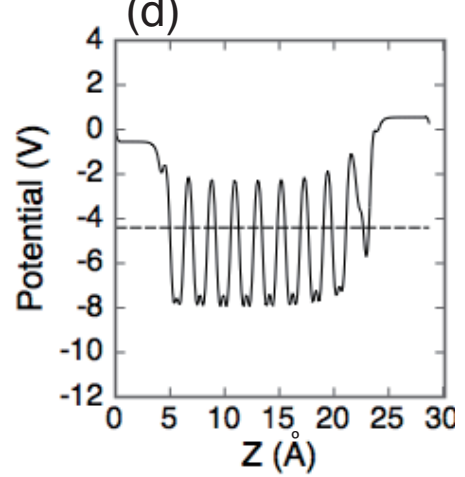

(f)

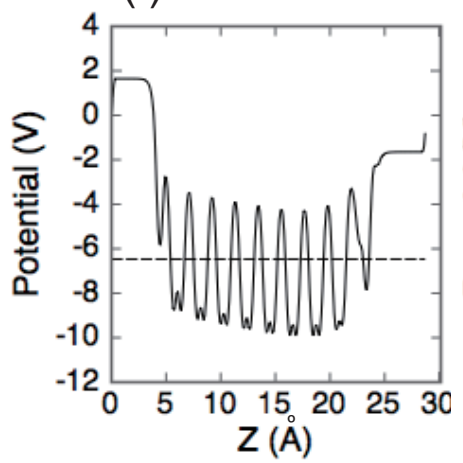

(h)

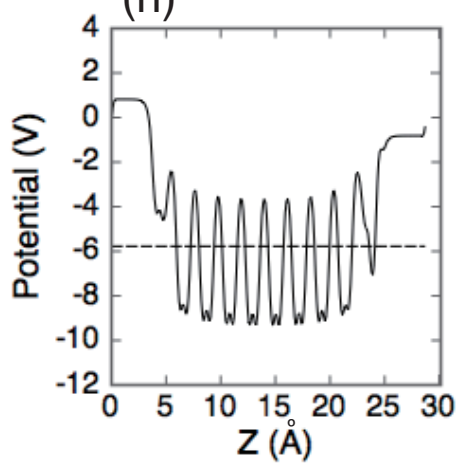

(b)

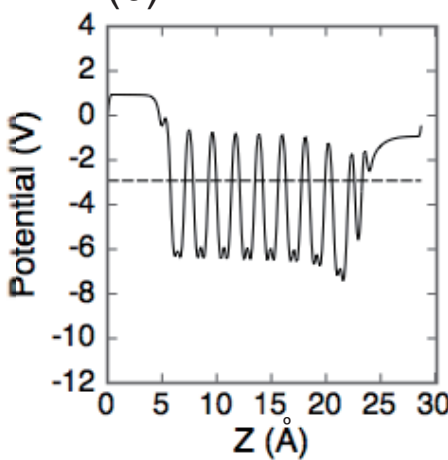

(d)

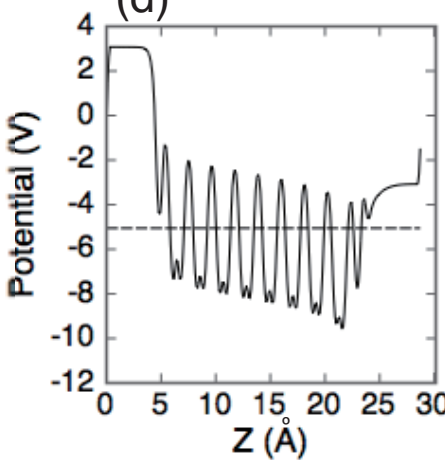

(g)

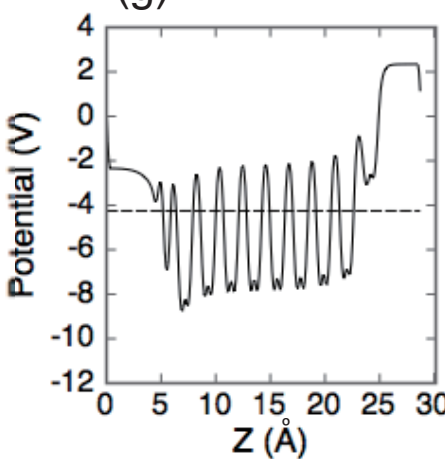

(c)

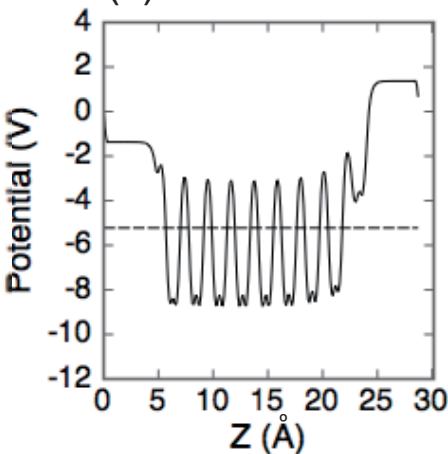

(e)

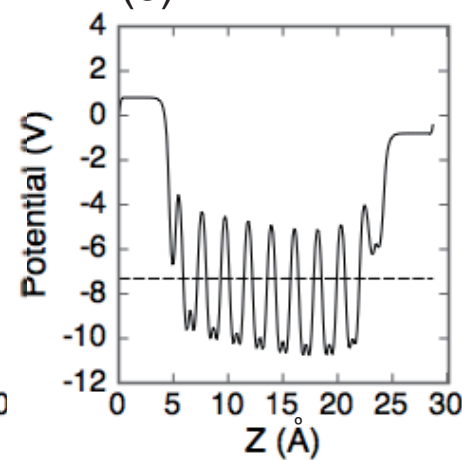

(g)

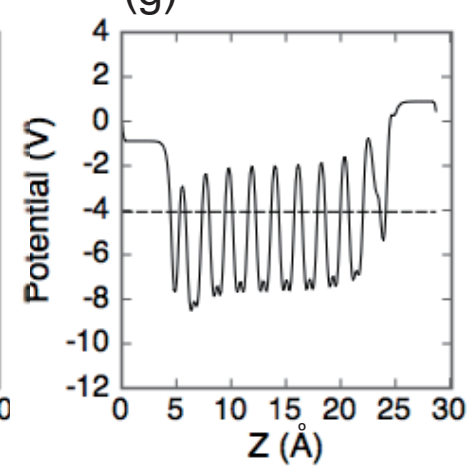

Fig. 5. Plane-averaged electrostatic potential across the graphene nanoribbons with zigzag edges asymmetrically functionalized by (a) $\mathrm{H}$ and ketone $(\mathrm{H} / \mathrm{O})$, (b) $\mathrm{H}$ and hydroxyl $(\mathrm{H} / \mathrm{OH}),(\mathrm{c}) \mathrm{H}$ and aldehyde $(\mathrm{H} / \mathrm{COH}),(\mathrm{d}) \mathrm{H}$ and carboxyl $(\mathrm{H} / \mathrm{COOH})$, (e) ketone and hydroxyl $(\mathrm{O} / \mathrm{OH})$, (f) ketone and aldehyde $(\mathrm{O} / \mathrm{COH}),(\mathrm{g})$ ketone and carboxyl $(\mathrm{O} / \mathrm{COOH})$, (f) hydroxyl and aldehyde $(\mathrm{OH} / \mathrm{COH})$, (g) hydroxyl and carboxyl $(\mathrm{OH} / \mathrm{COOH})$, and $(\mathrm{h})$ aldehyde and carboxyl $(\mathrm{COH} / \mathrm{COOH})$. Horizontal dotted lines indicate the Fermi level energy. The horizontal axis (z-axis) corresponds to the direction across the nanoribbons as shown in Fig. 1. 
functionalized by $\mathrm{H}$, ketone, aldehyde, and carboxyl groups. We found that the flat band at the Fermi level associated with the edge states is robust against edge functionalization. We also found that the NFE state substantially shifts downward and crosses the Fermi level for the nanoribbon functionalized by the hydroxyl groups. The work function difference obtained using the functional groups induces the polarity across the graphene nanoribbons, whose direction and amount are determined by the work function difference between two edges.

\section{Acknowledgements}

This work was supported by JST-CREST Grant Number JPMJCR1532 from the Japan Science and Technology Agency, JSPS KAKENHI Grant Numbers JP17H01069, JP16H00898, and JP16H06331 from the Japan Society for the Promotion of Science, and the Joint Research Program on Zero-Emission Energy Research, Institute of Advanced Energy, Kyoto University. Part of the calculations was performed on an NEC SX-Ace at the Cybermedia Center at Osaka University and on an SGI ICE XA/UV at the Institute of Solid State Physics, The University of Tokyo. 


\section{References}

1) M. S. Dresselhaus and G. Dresselhaus, Adv. Phys. 30139 (1981).

2) A. H. Castro Neto, F. Guinea, N. M. R. Peres, K. S. Novoselov and A. K. Geim, Rev. Mod. Phys. 81109 (2009).

3) A. A. Balandin, S. Ghosh, W. Bao, I. Calizo, D. Teweldebrhan, F. Miao, and C. N. Lau, Nano Lett. 8, 902 (2008).

4) S. Ghosh, I. Calizo, D. Teweldebrhan, E. P. Pokatilov, D. L. Nika, A. A. Balandin, W. Bao, F. Miao, and C. N. Lau, Appl. Phys. Lett. 92, 151911 (2008).

5) K. Saito, J. Nakamura, and A. Natori, Phys. Rev. B 76, 115409 (2007).

6) M. Morooka, T. Yamamoto, and K. Watanabe, Phys. Rev. B 77, 033412 (2008).

7) K. I. Bolotin, K. J. Sikes, Z. Jiang, M. Klima, G. Fudenberg, J. Hone, P. Kim, and H. L. Stormer, Solid State Commun. 146, 351 (2008).

8) J. B. Oostinga, H. B. Heersche, X. Liu, A. F. Morpurgo, and L. M. K. Vandersypen, Nat. Mater. 7, 151 (2007).

9) Y. Zhang, T. Tang, C. Girit, Z. Hao, M. C. Martin, A. Zettl, M. F. Crommie, Y. R. Shen, and F. Wang, Nature 459, 820 (2009).

10) K. S. Novoselov, A. K. Geim, S. V. Morozov, D. Jiang, Y. Zhang, S. V. Dubonos, I. V. Grigorieva, and A. A. Firsov, Science 306, 666 (2004).

11) K. S. Novoselov, A. K. Geim, S. V. Morozov, D. Jiang, M. I. Katsnelson, I. V. Grigorieva, S. V. Dubonos, and A. A. Firsov, Nature 438, 197 (2005).

12) Y. Zhang, Y. -W. Tan, H. L. Stormer, and P. Kim, Nature 438, 201 (2005).

13) J. B. Oostinga, H. B. Heersche, X. Liu, A. F. Morpurgo, and L. M. K. Vandersypen, Nat. Mater. 7151 (2007).

14) Y. Zhang, T. -T. Tang, C. Girit, Z. Hao, M. C. Martin, A. Zettl, M. F. Crommie, Y. R. Shen, and F. Wang, Nature 459, 820 (2009).

15) N. Shima, H. Aoki, Phys. Rev. Lett. 714389 (1993)

16) M. Maruyama, N. T. Cuong, and S. Okada, Carbon 109, 755 (2016).

17) M. Fujita, K. Wakabayashi, K. Nakada, and K. Kusakabe, J. Phys. Soc. Jpn. 65, 1920 (1996).

18) K. Nakada, M. Fujita, G. Dresselhaus, and M.S. Dresselhaus, Phys. Rev. B 54, 17954 (1996).

19) A. Yamanaka and S. Okada, Carbon 96, 351 (2016).

20) F. Cervantes-Sodi, G. Csányi, S. Piscanec, and A. C. Ferrari, Phys. Rev. B 77, 
165427 (2008).

21) T. Kuila, S. Bose, A. K. Mishra, P. Khanra, N. H. Kim, J. H. Lee, Prog. Mater. Sci. 57, 1061(2012).

22) R. Sekiya, Y. Uemura, H. Murakami, and T. Haino, Angew. Chem. 535619 (2014).

23) R. Taira, A. Yamanaka, and S. Okada, Appl. Phys. Express 9, 115102 (2016).

24) S. Okada, M. Igami, K. Nakada, and A. Oshiyama, Phys. Rev. B 62, 9896 (2000).

25) M. Maruyama and S. Okada, J. Phys. Chem. C 120, 1293 (2016).

26) M. Posternak, A. Baldereschi, A. J. Freeman, E. Wimmer, and M. Weinert, Phys. Rev. Lett. 50, 761 (1983).

27) M. Posternak, A. Baldereschi, A. J. Freeman, and E. Wimmer, Phys. Rev. Lett. 52, 863 (1984).

28) A. Catellani, M. Posternak, A. Baldereschi, and A. J. Freeman, Phys. Rev. B 36, 6105 (1987).

29) X. Blase, A. Rubio, S. G. Louie, and M. L. Cohen, Phys. Rev. B 51, 6868 (1995).

30) N.-T. Cuong, M. Otani, and S. Okada, J. Phys. Cond. Matter 26, 135001 (2014).

31) Y. Miyamoto, A. Rubio, X. Blase, M. L. Cohen, and S. G. Louie, Phys. Rev. Lett. 74, 2993 (1995).

32) S. Okada, A. Oshiyama, and S. Saito, Phys. Rev. B 62, 7634 (2000).

33) X. Blase, A. Rubio, S.G. Louie, and M.L. Cohen, Europhys. Lett. 28, 335 (1994).

34) Q. Liu, Z. Li, and J. Yang, Chinese J. Chem. Phys. 24, 22 (2011).

35) A. Yamanaka and S. Okada, Appl. Phys. Express 7, 125103 (2014).

36) A. Yamanaka and S. Okada, Sci. Rep. 6, 30653 (2016).

37) P. Hohenberg and W. Kohn, Phys. Rev. 136, B864 (1964).

38) W. Kohn and L. J. Sham, Phys. Rev. 140, A1133 (1965).

39) Y. Morikawa, K. Iwata, and K. Terakura, Appl. Surf. Sci. 169-170, 11 (2001).

40) J. P. Perdew and A. Zunger, Phys. Rev. B 23, 5048 (1981).

41) D. M. Ceperley and B. J. Alder, Phys. Rev. Lett. 45, 566 (1980).

42) D. Vanderbilt, Phys. Rev. B 41, 7892 (1990).

43) M. Otani and O. Sugino, Phys. Rev. B 73, 115407 (2006). 